\section{Check for updates}

Cite this: Soft Matter, 2018, 14,9436

Received 28th August 2018, Accepted 6th November 2018 DOI: $10.1039 / c 8 s m 01765 d$

rsc.li/soft-matter-journal

\title{
Electrostatic interaction of particles trapped at fluid interfaces: effects of geometry and wetting properties
}

\author{
Arghya Majee, (D) *ab Markus Bier (D)*abc and S. Dietrich ${ }^{\mathrm{ab}}$
}

\begin{abstract}
The electrostatic interaction between pairs of spherical or macroscopically long, parallel cylindrical colloids trapped at fluid interfaces is studied theoretically for the case of small inter-particle separations. Starting from the effective interaction between two planar walls and by using the Derjaguin approximation, we address the issue of how the electrostatic interaction between such particles is influenced by their curvatures and by the wetting contact angle at their surfaces. Regarding the influence of curvature, our findings suggest that the discrepancies between linear and nonlinear Poisson-Boltzmann theory, which have been noticed before for planar walls, also occur for spheres and macroscopically long, parallel cylinders, though their magnitude depends on the wetting contact angle. Concerning the influence of the wetting contact angle $\theta$ simple relations are obtained for equally sized particles which indicate that the inter-particle force varies significantly with $\theta$ only within an interval around $90^{\circ}$. This interval depends on the Debye length of the fluids and on the size of the particles but not on their shape. For unequally sized particles, a more complicated relation is obtained for the variation of the inter-particle force with the wetting contact angle.
\end{abstract}

\section{Introduction}

Colloidal particles trapped at a fluid interface usually adopt configurations which are energetically more favorable compared to those occurring in the adjacent bulk phase(s). ${ }^{1,2}$ This can be exploited for a wide spectrum of systems ranging from micrometer down to nanometer in size and from biological to industrial processes, including the stabilization of Pickering emulsions, ${ }^{3}$ the transport of drugs and nutrients in biological systems, ${ }^{4}$ the formation of artificial cells, ${ }^{5}$ oil recovery, water purification, mineral processing, maintaining proper foaminess of cosmetic and food products, ${ }^{2}$ and the fabrication of various nanostructured devices. ${ }^{6,7}$ The trapping phenomenon depends on the wetting properties, the size, and the shape of the colloidal particles, because it hinges on the particlemediated reduction of the fluid-fluid interfacial area, and consequently on the net reduction of the free energy of the system. $^{2}$

\footnotetext{
${ }^{a}$ Max-Planck-Institut für Intelligente Systeme, Heisenbergstr. 3, 70569 Stuttgart, Germany.E-mail: majee@is.mpg.de, bier@is.mpg.de

${ }^{b} I V$. Institut für Theoretische Physik, Universität Stuttgart, Pfaffenwaldring 57 , 70569 Stuttgart, Germany

${ }^{c}$ Fakultät Angewandte Natur- und Geisteswissenschaften, Hochschule für angewandte Wissenschaften Würzburg-Schweinfurt, Ignaz-Schön-Str. 11, 97421 Schweinfurt, Germany
}

On a mesoscopic level, the wetting properties of a colloidal particle are described best by the contact angle $\theta$ of the fluidfluid interface with respect to the particle surface (see Fig. 1). Following the standard convention, we measure $\theta$ inside the more polar phase. For oil-water systems this implies that a particle is hydrophilic if $\theta<90^{\circ}$, hydrophobic if $\theta>90^{\circ}$, and neutrally wetted for $\theta=90^{\circ}$. Within the continuum model, the equilibrium contact angle $\theta$ of a particle is determined solely by energies associated with the three interfaces (two particle-liquid and one liquid-liquid) according to the well-known Young equation. ${ }^{8,9}$ In general, particles, which are partially wetted by both fluid phases, attach most stably to an interface because the corresponding trapping energy often exceeds several orders of $k_{\mathrm{B}} T .^{2,10}$

Wetting properties are crucial not only for the adsorption of a single particle at an interface, but also for the interaction between several of them. For example, whereas very hydrophobic silica particles $\left(\theta \geq 129^{\circ}\right)$ form well-ordered monolayer structures (with inter-particle separations of several particle diameter) at octane-water interfaces, less hydrophobic $\left(\theta \leq 115^{\circ}\right)$ particles fail to do so. ${ }^{11,12}$ This can be attributed to different strengths of the repulsive electrostatic force, which acts mainly through the oil-phase because the electrostatic field is well screened inside the aqueous phase at the high salt concentrations used. Moreover, the capillary interaction due to the overlap of the interface deformation field around each particle also 


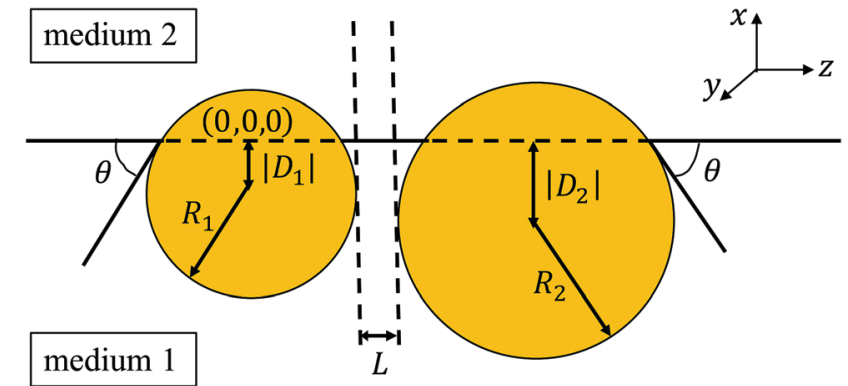

Fig. 1 Cross-sectional view in the $y=0$ plane of a system with two spherical or parallel cylindrical colloids (yellow discs) floating at a fluid interface indicated by the horizontal line. The projection of the center of the left particle onto the interface is chosen as the center $(0,0,0)$ of the Cartesian coordinate system used to describe the system. The fluid medium below the interface is called medium " 1 " and the one above the interface medium " 2 ". The particles are chemically identical, such that the contact surfaces with one of the media carry the same charge densities and the same wetting contact angles $\theta$. The particles may differ in size with radius $R_{1}$ on the left and $R_{2}$ on the right particle. The horizontal distance of the particles is characterized by the width $L$ of the gap between both particles as depicted by the vertical dashed lines. The equilibrium heights of the centers of the left and right particles from the interface are given by $\left|D_{1}\right|$ and $\left|D_{2}\right|$, respectively, with $\theta \in[0, \pi]$ determining the sign of $D_{1}$ and $D_{2}$ according to the relations $D_{1}=-R_{1} \cos \theta$ and $D_{2}=-R_{2} \cos \theta$

depends on the contact angle $\theta$. However, here we disregard deformations of the interface, which can be significant if the particle surfaces are rough ${ }^{13}$ or the particles are large (radius $\gtrsim$ $10 \mu \mathrm{m}),{ }^{14-19}$ and we focus only on the electrostatic interaction between the colloids.

Whereas the electrostatic interaction between particles trapped at a fluid interface has been studied extensively since the pioneering studies by Pieranski ${ }^{20}$ and Hurd, ${ }^{21}$ most of the investigations deal with the case of particles situated far away from each other. At long distances the electrostatic pairinteraction takes the particularly simple form of an interaction between two electric dipoles, which are generated by the asymmetric counterion distribution at the particle surfaces in contact with the two fluid phases. It has been shown that in this case the linearization of the Poisson-Boltzmann (PB) theory is applicable. ${ }^{21}$ Recent studies, directed towards the opposite limit of small inter-particle separations, have been performed within the linearized PB theory ${ }^{22-24}$ or by considering a flat plate geometry ${ }^{22,24,25}$ in order to simplify the problem. Whereas the former approximation is often violated at short separations, the latter represents the ideal situation of a contact angle of exactly $90^{\circ}$ and the absence of particle curvature. However, in reality, the contact angle $\theta$ can vary significantly to either side of $90^{\circ 2,10,19,26}$ and whether the particle curvature plays any important role at short inter-particle separations still remains to be addressed within the nonlinear PB theory.

Accordingly, in this contribution, we investigate the electrostatic interaction between spherical and parallel cylindrical colloids with an arbitrary contact angle appearing at a fluid interface. As long as the size of the particles is sufficiently larger than both the length scale of the interaction and the inter-particle separation, which is usually the case for short inter-particle separations we are interested in, one can apply the Derjaguin approximation (DA) using results of the corresponding case of planar walls. ${ }^{27}$ Having recently solved this two-plate problem exactly (i.e., without using the superposition approximation) within the nonlinear PB theory, ${ }^{25}$ we proceed one step further and compute the force between a pair of spheres or parallel cylinders. A similar approach has already been employed and proved to be valid in this context. ${ }^{28,29}$ However, the present study differs from those treatments in several aspects. Whereas ref. 28 deals with macroscopically long cylinders trapped at an oil-water interface and having a constant surface potential, which is most suitable for metallic particles, we consider dielectric particles described by constant charge densities at their surfaces. Moreover, to keep our analysis general and to be consistent with experimental observations, ${ }^{30-34}$ we consider the particle surfaces to be charged in both fluid phases, which is not the case in ref. 28. On the other hand, ref. 29 describes the interaction between spherical particles by using the superposition approximation, which has been shown to be qualitatively wrong for small inter-particle separations ${ }^{22}$ and it discards any interaction between the particle-water surface of one particle and the particle-oil surface of the other particle. As explained in the next section, this latter contribution to the interaction energy is included in our calculation via the line contribution.

\section{Model and formalism}

As depicted in Fig. 1 , we consider two particles with radii $R_{1}$ and $R_{2}$ placed at a fluid-fluid interface described by a threedimensional Cartesian coordinate system. The projection of the center of the left particle onto the interface is chosen as the origin $(0,0,0)$ of the coordinate system. The particles are either two spheres or two macroscopically long, parallel cylinders with axes in $y$-direction; their cross-sections in the plane $y=0$ are shown in Fig. 1. The fluid-fluid interface is indicated by the horizontal line at $x=0$. Although the particles can differ in size, they are taken to be chemically identical such that the surfaces of both particles in contact with the same fluid phase carry the same surface charge density, and that the contact angle $\theta$ is the same for both particles. This is a simplifying assumption because chemically identical particles in general need not to be equally charged. ${ }^{35}$ In equilibrium the centers of the left and the right particle are located at $x=D_{1}$ and $x=D_{2}$, respectively. Depending upon the contact angle $\theta \in[0, \pi]$, both $D_{1}=-R_{1} \cos \theta$ and $D_{2}=-R_{2} \cos \theta$ can be negative (for $\theta<\frac{\pi}{2}$; the case considered in Fig. 1) as well as positive (for $\theta>\frac{\pi}{2}$ ). In between the particles a gap of width $L$ occurs (see the vertical dashed lines in Fig. 1) so that the horizontal center-to-center distance is $L+R_{1}+R_{2}$. The fluid phase below (above) the interface occupying the half-space $x<0(x>0)$ is denoted by medium " 1 " ("2"). Both fluids are modeled as structureless, continuous media with dielectric constant $\varepsilon_{i}=\varepsilon_{\mathrm{r}, i} \varepsilon_{0}, i \in\{1,2\}$, where $\varepsilon_{\mathrm{r}, i}$ is the relative permittivity of medium $i$ and $\varepsilon_{0}$ is the vacuum permittivity. The ionic strength of added salt in medium $i \in\{1,2\}$ is denoted by $I_{i}$. 
The corresponding Debye screening length in each medium is given by $\kappa_{i}^{-1}=\sqrt{\varepsilon_{\mathrm{r}, i} /\left(8 \pi \ell_{\mathrm{B}} I_{i}\right)}$ where $\ell_{\mathrm{B}}=e^{2} /\left(4 \pi \varepsilon_{0} k_{\mathrm{B}} T\right)$ is the vacuum Bjerrum length with $e>0, k_{\mathrm{B}}$, and $T$ being the elementary charge, the Boltzmann constant, and the absolute temperature, respectively. Within our description at the mean-field level, the length scale of interest is the Debye length as both the local charge density and the electrostatic interaction vary on this scale. Phenomena which occur on smaller length scales, e.g., the structuring of liquids on the molecular length scale, are not considered. Here we consider medium "2" to be the less polar phase in the sense that $\kappa_{2}{ }^{-1}>$ $\kappa_{1}^{-1}$. With this the criteria for the applicability of the DA are that both radii $R_{1}$ and $R_{2}$ have to be much larger than $\kappa_{2}{ }^{-1}$ and $L$.

Within the DA, one basically decomposes the two interacting particles into infinitesimal surface elements. Assuming that the elementary surface pieces, which face each other, interact like flat parallel surfaces, the total interaction between the two curved objects is obtained via integration over the whole surface. Here, however, the particle surfaces are homogeneously charged only separately inside each medium, the properties of which in general differ. As a result, a three-phase contact line is formed where a particle surface intersects the fluid interface; two such contact lines on opposing particles interact as well. But this does not introduce any additional constraint for applying the DA. In the spirit of the DA, each of these two contact lines can be divided in infinitesimal pieces and the total contribution due to the line interaction can be obtained as long as the interaction between two parallel lines is known. Therefore, in order to apply the DA, one needs to know the interaction of parallel flat surfaces dipped into medium " 1 " or medium " 2 " and the interaction between two parallel three-phase contact lines. These are exactly the quantities we calculated in ref. 25 numerically by solving the nonlinear PB equation. To be more precise, the relevant quantities, as defined in ref. 25 , are $\omega_{\gamma, i}(r)$, which is the interaction energy per total surface area between two parallel, planar surfaces dipped at a distance $r$ into medium $i \in\{1,2\}$, and $\omega_{\tau}(r)$, which is the interaction energy per total line length between two parallel three-phase contact lines at a distance $r$. Please note that the interaction of the surface of one particle in contact with medium " 1 " and that of the other particle in contact with medium " 2 " is included in the line contribution $\omega_{\tau}(r)$. In order to tackle the problem efficiently, we first fit simple functions to the numerical data for $\omega_{\gamma, 1}(r), \omega_{\gamma, 2}(r)$, and $\omega_{\tau}(r)$ obtained by full minimization of the nonlinear PB grand potential. It turns out that a reasonably good fitting can be obtained by superposing exponential contributions as follows:

$$
\begin{aligned}
& \omega_{\gamma, 1}(r)=\sum_{i=1}^{3} a_{i} \exp \left(-b_{i} r\right), \\
& \omega_{\gamma, 2}(r)=\sum_{i=1}^{3} c_{i} \exp \left(-d_{i} r\right),
\end{aligned}
$$

and

$$
\omega_{\tau}(r)=\sum_{i=1}^{4} g_{i} \exp \left(-h_{i} r\right) .
$$

For two flat plates, all interactions decay exponentially in the limit of large separations: $\omega_{\gamma, i}(r \rightarrow \infty) \sim \exp \left(-\kappa_{i} r\right)$ and $\omega_{\tau}(r \rightarrow \infty) \sim \exp \left(-\kappa_{2} r\right)$ (note the convention $\kappa_{2}{ }^{-1}>\kappa_{1}{ }^{-1}$ ). As a result, when fitting the data over a sufficiently large interval of $r$ (i.e., a few Debye lengths), the slowest of the decay rates $b_{i}$ in eqn (1) equals $\kappa_{1}$ and the slowest of the decay rates $d_{i}$ in eqn (2) as well as the slowest of the decay rates $h_{i}$ in eqn (3) equal $\kappa_{2}$. All three interactions, i.e., $\omega_{\gamma, 1}, \omega_{\gamma, 2}$, and $\omega_{\tau}$ result in forces onto the particles, which can be obtained by taking the negative derivative with respect to the appropriate distance between the facing surface or line elements, followed by integrating over the particles. Due to the geometry of the problem, the electrostatic force between the particles acts only in the horizontal $z$-direction. Please note that the movement of the particles in the vertical $x$-direction is suppressed by the steep and strong trapping potential. ${ }^{2}$ In the following, we denote the $z$-component of the electrostatic force, which the left particle in Fig. 1 exerts on the right one, by $F(L)$, and we decompose it, according to $F(L)=F_{1}(L)+F_{2}(L)+F_{3}(L)$, into the surface contribution $F_{1}(L)$ due to the surface interaction $\omega_{\gamma, 1}$ in medium " 1 ", the surface contribution $F_{2}(L)$ due to the surface interaction $\omega_{\gamma, 2}$ in medium " 2 ", and the line contribution $F_{3}(L)$ due to the line interaction $\omega_{\tau}$.

\section{Results and discussion}

In this section we discuss the variation of the force $F(L)$ between the particles as function of the particle sizes (radii $R_{1}$ and $R_{2}$ ), the particle separation $L$, and the contact angle $\theta$. For our discussion we consider two typical experimental setups and in each case the results for a pair of spheres as well as a pair of parallel cylinders are presented. Between the two systems considered below, the data for flat wall interactions for water-lutidine interfaces are taken from ref. 25 and those for water-octanol interfaces are newly generated here. We mention that all numerical examples presented here have been chosen such that the conditions for applying the DA are satisfied. Consequently, systems featuring oil with very low dielectric constants, such as decane or octane, have been excluded because the corresponding Debye length $\kappa_{2}{ }^{-1}$ is too large for them to satisfy the condition $R_{1}, R_{2} \gg \kappa_{2}{ }^{-1}$, even for micron size colloids.

\section{A. Water-lutidine interface}

First, we consider a system consisting of polystyrene particles placed at a water-lutidine (2,6-dimethylpyridine) interface at temperature $T=313 \mathrm{~K}$. The added salt is NaI with bulk ionic strengths $I_{1}=1 \mathrm{mM}$ and $I_{2}=0.85 \mathrm{mM}$. The relative permittivities are $\varepsilon_{\mathrm{r}, 1}=72$ for the water-rich phase (medium " 1 ") and $\varepsilon_{r, 2}=62$ for the lutidine-rich phase (medium " 2 "). The chemically identical particles are assumed to be similarly charged; the magnitude of the surface charge density in contact with the aqueous phase is $\sigma_{1}=0.1 e \mathrm{~nm}^{-2}$ and that in contact with the oil-phase is $\sigma_{2}=0.01 e \mathrm{~nm}^{-2}$. Differences in the solubilities of the ions in the two fluids result in a potential difference between the bulk of the two media, which is called the Donnan 
potential or Galvani potential difference, ${ }^{36}$ and which, for our system, is assumed to be $1 k_{\mathrm{B}} T / e$. These numbers correspond to a standard set of parameters as used in ref. 25. They are either taken or estimated from various experimental studies. ${ }^{31,37-42}$

1. Spheres. In the case of two interacting spheres at a fluid interface, after performing the surface and line integrations, for the three distinct, lateral force contributions the following expressions are obtained:

$$
\begin{aligned}
F_{1}(L)= & \sum_{i=1}^{3} \frac{\pi a_{i}}{\left(\frac{1}{R_{1}}+\frac{1}{R_{2}}\right)} \exp \left\{-b_{i}\left(L+\frac{\left(D_{1}-D_{2}\right)^{2}}{2\left(R_{1}+R_{2}\right)}\right)\right\} \\
& \times\left[1-\operatorname{erf}\left(\left(D_{1} R_{2}+D_{2} R_{1}\right) \sqrt{\frac{b_{i}}{2 R_{1} R_{2}\left(R_{1}+R_{2}\right)}}\right)\right], \\
F_{2}(L)= & \sum_{i=1}^{3} \frac{\pi c_{i}}{\left(\frac{1}{R_{1}}+\frac{1}{R_{2}}\right)} \exp \left\{-d_{i}\left(L+\frac{\left(D_{1}-D_{2}\right)^{2}}{2\left(R_{1}+R_{2}\right)}\right)\right\} \\
& \times\left[1+\operatorname{erf}\left(\left(D_{1} R_{2}+D_{2} R_{1}\right) \sqrt{\frac{d_{i}}{2 R_{1} R_{2}\left(R_{1}+R_{2}\right)}}\right)\right],
\end{aligned}
$$

where $\operatorname{erf}(x)$ denotes the error function, ${ }^{46}$ and

$$
F_{3}(L)=\sum_{i=1}^{4} g_{i} \sqrt{\frac{2 \pi h_{i}}{\left(\frac{1}{R_{1}}+\frac{1}{R_{2}}\right)}} \exp \left\{-h_{i}\left(L+\frac{D_{1}^{2}}{2 R_{1}}+\frac{D_{2}^{2}}{2 R_{2}}\right)\right\}
$$

with $D_{1}=-R_{1} \cos \theta$ and $D_{2}=-R_{2} \cos \theta$. Variations of the total force $F(L)=F_{1}(L)+F_{2}(L)+F_{3}(L)$ in the units of $10^{3} \kappa_{1} / \beta$ with the scaled separation $\kappa_{1} L$ for different system parameters are shown in Fig. 2. Here $\beta=1 /\left(k_{\mathrm{B}} T\right)$ is the inverse thermal energy. As one can infer from Fig. 2(a), for equally-sized spheres $\left(R_{1}=R_{2}=R\right)$ with a contact angle of $\theta=90^{\circ}$, the effective force scales linearly with the size of the particles and decays exponentially with increasing separation between the particles. Both the linear scaling with $R$ and the exponential decay with $L$ can be directly inferred from the inset of Fig. 2(a) where the ratio of the dimensionless force $\beta F / \kappa_{1}$ to the scaled radius $\kappa_{1} R$ is plotted as a function of the scaled separation $\kappa_{1} L$ using a semilogarithmic scale, revealing data collapse. The exponential decay is expected to occur in the sense that all effective interactions decay exponentially for a pair of interacting flat plates, which remains unaffected while using the DA, and the linear scaling with $R$ is a direct consequence of the DA. Fig. 2(b and c) display the variation of the scaled force with contact angle $\theta$ for equally-sized particles. As one can see, for $\kappa_{1} R \approx$ 100 (b), the force increases with decreasing contact angle but de facto it varies only within the interval $80^{\circ}<\theta<100^{\circ}$. A similar phenomenon is observed for $\kappa_{1} R \approx 30$ (c) albeit with variation in a slightly broader interval $75^{\circ}<\theta<105^{\circ}$. Fig. 2 (d and e) show the variation of the force with contact angle $\theta$ as function of separation distance $\kappa_{1} L$ for unequally-sized spheres. For the relatively small size-asymmetry in Fig. 2(d), the force between the particles can increase in a certain range of separation between the particles as the contact angle $\theta$ is decreased slightly from $90^{\circ}$ downwards. Otherwise the force is weaker than the one obtained for a neutral wetting situation $\theta=90^{\circ}$. For the larger size-asymmetry in Fig. 2(e), the force becomes weaker as soon as the contact angle $\theta$ differs from $90^{\circ}$. However, in both cases, as considered in Fig. 2(d and e), the force between the particles decreases upon increasing $\theta$ in the interval $\theta>90^{\circ}$. Finally, in Fig. 2(f), we compare the force obtained within the linearized and the nonlinear PB theory for equally sized spheres. As one can see from the plot, the forces differ by almost an order of magnitude even at large separations such as $\kappa_{1} L \approx 6$ for $\theta=90^{\circ}$. It turns out that with increasing contact angle $\theta$ this difference diminishes; see the inset of Fig. 2(f), where both curves are of almost the same magnitude. This is expected since the portion of the particles immersed in the more polar phase, for which the electrostatic interaction is stronger because $\kappa_{1} \approx \kappa_{2}\left[\kappa_{1} \approx 0.1059 \mathrm{~nm}^{-1}, \kappa_{2} \approx 0.1053 \mathrm{~nm}^{-1}\right]$ and $\sigma_{1} \gg \sigma_{2}$, decreases with increasing contact angle. Note that this situation differs from the one in ref. 11 and 12 in which $\kappa_{1} \gg \kappa_{2}$. A similar discrepancy appeared while comparing the interactions within the linear and nonlinear theory for parallel flat surfaces. ${ }^{25}$ Thus taking into account particle curvature does not significantly change this result.

All these observations are in accordance with the force expressions given in eqn (4)-(6). For micron-sized particles considered here, in most of the cases the line contribution to the total interaction is negligible. Therefore, the total force $F(L)$ is dominated by the surface contributions $F_{1}(L)$ (eqn (4)) and $F_{2}(L)$ (eqn (5)). For $\theta=90^{\circ}$, which is the case considered in Fig. 2(a), one has $D_{1}=D_{2}=0$. Moreover, with $R_{1}=$ $R_{2}=R$, eqn (4) and (5) reduce to $F_{1}(L)=\frac{\pi R}{2} \sum_{i=1}^{3} a_{i} \exp \left(-b_{i} L\right)$ and $F_{2}(L)=\frac{\pi R}{2} \sum_{i=1}^{3} c_{i} \exp \left(-d_{i} L\right)$, respectively, which transparently explain the linear variation of the force with the particle size $R$ (the coefficients $a_{i}$ and $c_{i}$ do not depend on $R$ ) and its exponential decay as function of the separation $L$. The decay rate in the limit of large distances is determined by the smaller of the two Debye lengths $\kappa_{1}{ }^{-1}$ and $\kappa_{2}{ }^{-1}$ (please note that for our system $\kappa_{1}{ }^{-1} \approx \kappa_{2}{ }^{-1}$ ). For $R_{1}=R_{2}=R$ with an arbitrary contact angle $\theta$, one has $D_{1}-D_{2}=0$ so that the dependence on $\theta$ appears in eqn (4) and (5) only through the terms involving the error functions, which reduce to $1-\operatorname{erf}\left(-\cos \theta \sqrt{b_{i} R}\right)$ and $1+\operatorname{erf}\left(-\cos \theta \sqrt{d_{i} R}\right)$, respectively. Since the error function levels off to $|\operatorname{erf}(x)| \approx 1$ for $|x| \gtrsim 2$, the variation of the force with respect to $\theta$ in eqn (4) saturates once the slowest decay rate $b_{i}$, which in the present case is $\kappa_{1}$, satisfies the inequality

$$
|\cos \theta| \approx \frac{2}{\sqrt{\kappa_{1} R}} .
$$

Similarly, in eqn (5) the saturation is obtained once the slowest decay rate $d_{i}$, i.e., $\kappa_{2}$ satisfies the inequality

$$
|\cos \theta| \gtrsim \frac{2}{\sqrt{\kappa_{2} R}} .
$$



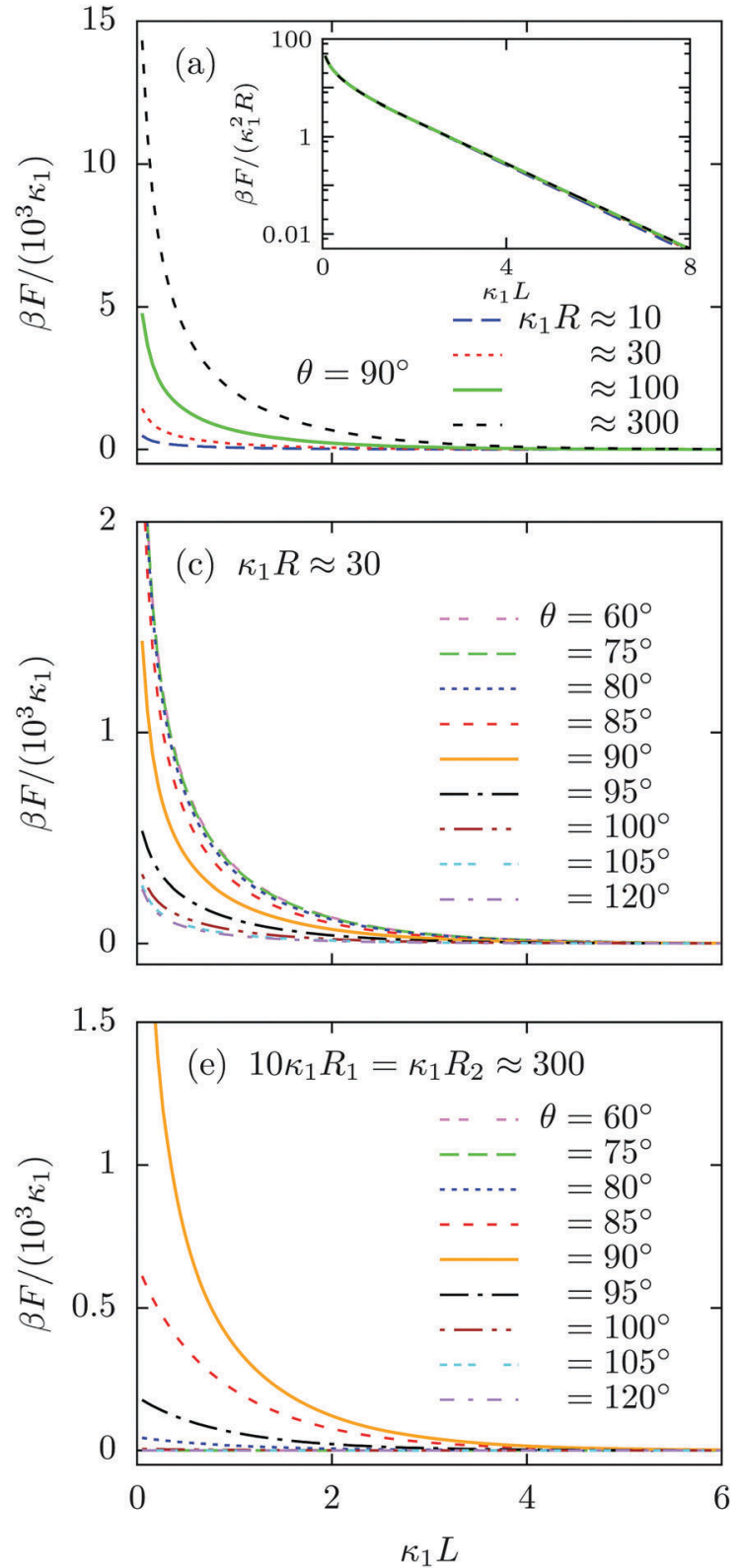
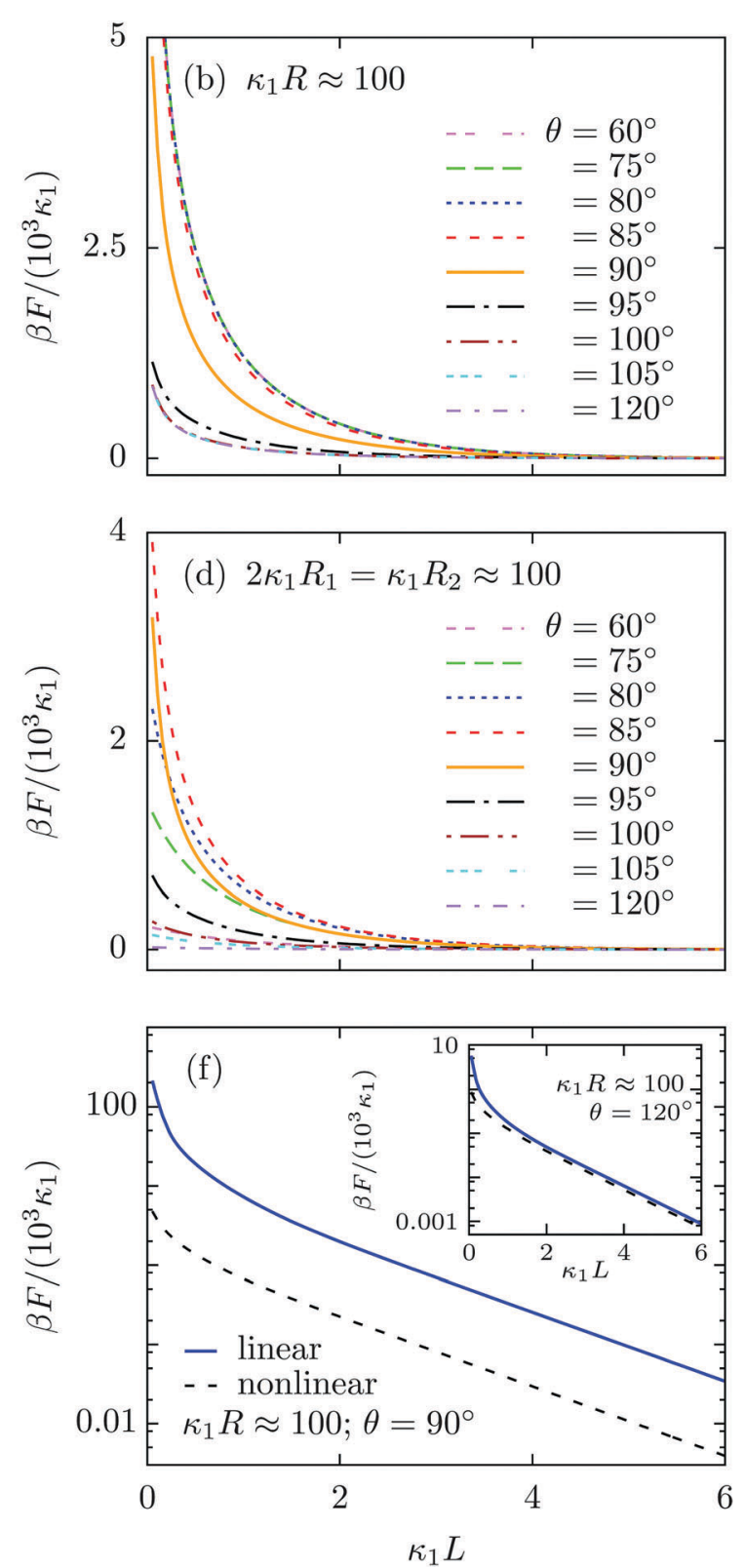

Fig. 2 Variation of the lateral component of the force $F(L)$ due to the electrostatic interaction between a pair of spherical colloidal particles, expressed in units of $10^{3} \kappa_{1} / \beta$, as function of their scaled separation $\kappa_{1} L$ for (a) equally sized $\left(R_{1}=R_{2}=R\right)$ spheres of varying radius with contact angle $\theta=90^{\circ}$, (b) equally sized $\left(\kappa_{1} R \approx 100\right)$ particles with varying $\theta$, (c) equally sized $\left(\kappa_{1} R \approx 30\right)$ particles with varying $\theta$, (d) unequally sized $\left(2 \kappa_{1} R_{1}=\kappa_{1} R_{2} \approx 100\right)$ particles with varying $\theta$, (e) unequally sized $\left(10 \kappa_{1} R_{1}=\kappa_{1} R_{2} \approx 300\right)$ particles with varying $\theta$, and (f) equally sized $\left(\kappa_{1} R \approx 100\right)$ particles with $\theta=90^{\circ}$ within linear and nonlinear PB theory. As shown by panel (a) and its inset, the force increases linearly with increasing $R$ and decays exponentially with increasing separation $\kappa_{1} L$. Panels (b) and (c) suggest that, for equally sized spheres, the force increases significantly with decreasing contact angle $\theta$ only within an interval around $90^{\circ}$. Outside this interval the force remains de facto constant and the interval of $\theta$, across which the force actually varies, widens upon decreasing $\kappa_{1} R$. For unequally sized spheres, if the size asymmetry is moderate, the force may increase as well as decrease if the contact angle deviates from $90^{\circ}$ (panel (d)). However, if the size contrast is high, the force becomes weaker once $\theta$ is slightly shifted away from $90^{\circ}$ in either direction (panel (e)). From panel (f) and the inset therein one can infer that the discrepancy between the linear and the nonlinear results diminishes with increasing $\theta$.

Note that once the slowest decay rates $b_{i}$ and $d_{i}$ satisfy these conditions, all the other decay rates will do so, too. For $\kappa_{1} R \approx$ 100 (and therefore, $\kappa_{2} R \approx 100$ as $\kappa_{1} \approx \kappa_{2}$ ), both eqn (7) and (8) predict that the force varies appreciably only within the interval $78^{\circ} \leq \theta \leq 102^{\circ}$, which one precisely observes in Fig. 2(b). Decreasing the contact angle $\theta$ from $\frac{\pi}{2}$ implies that the particles become more hydrophilic. Consequently, the contribution $F_{1}(L)$ to the total force increases while $F_{2}(L)$ decreases upon decreasing $\theta$. Finally, at $\theta \approx 78^{\circ}$ the former attains a non-zero finite value and the latter vanishes (please note the different signs in front of the error functions in eqn (4) and (5)). On the other hand, increasing the contact angle $\theta$ from $\frac{\pi}{2}$ implies that the 
particles become more hydrophobic. As a result, the contribution $F_{2}(L)$ increases and $F_{1}(L)$ decreases with the former attaining a non-zero finite value while the latter is vanishing at $\theta \approx 102^{\circ}$. Upon decreasing $\kappa_{1} R$, the interval of $\theta$ over which the force varies broadens as can be inferred from Fig. 2(c). For unequal particles sizes, i.e., for $R_{1} \neq R_{2}$, the dependence on $\theta$ in eqn (4) and (5) originate from both the exponential and the error function. For moderate size-asymmetry, like the one considered in Fig. 2(d), a competition between these two functions determines the variation of the force with $\theta$. However, for the extremely asymmetric case considered in Fig. 2(e), the difference $D_{1}-D_{2}$ is large and the exponential terms dominate as soon as $\theta$ differs slightly from $90^{\circ}$.
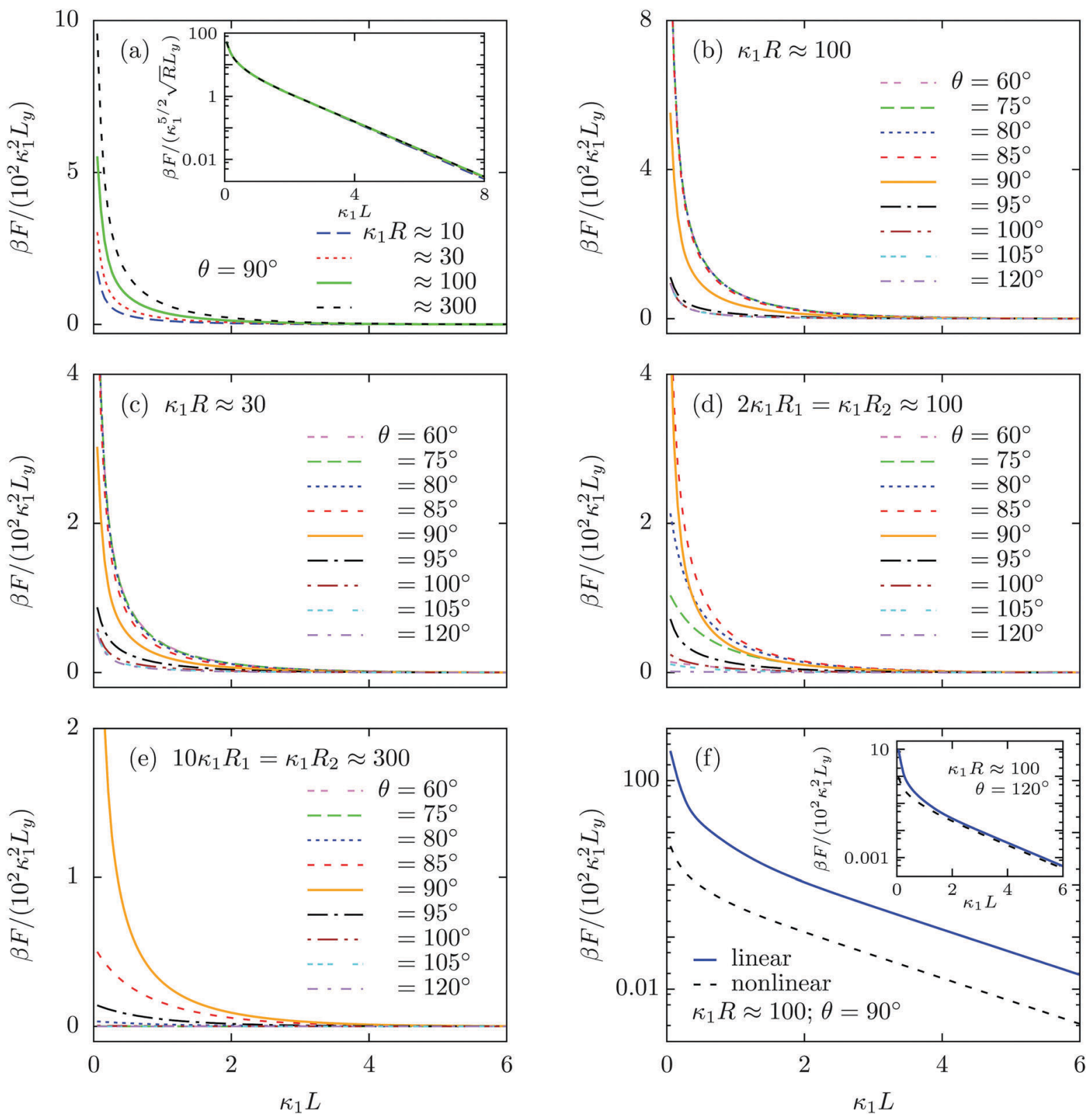

Fig. 3 Variation of the $z$-component (see Fig. 1) of the force $F(L)$, per length $L_{y}$ in the $y$-direction, due to the electrostatic interaction between a pair of parallel cylindrical colloids, expressed in units of $10^{2} \kappa_{1}^{2} / \beta$, as function of their scaled separation $\kappa_{1} L$ for (a) equally sized $\left(R_{1}=R_{2}=R\right)$ cylinders of varying radius with contact angle $\theta=90^{\circ}$, (b) equally sized $\left(\kappa_{1} R \approx 100\right)$ particles with varying $\theta$, (c) equally sized $\left(\kappa_{1} R \approx 30\right)$ particles with varying $\theta$, (d) unequally sized $\left(2 \kappa_{1} R_{1}=\kappa_{1} R_{2} \approx 100\right)$ particles with varying $\theta$, (e) unequally sized $\left(10 \kappa_{1} R_{1}=\kappa_{1} R_{2} \approx 300\right)$ particles with varying $\theta$, and (f) equally sized ( $\left.\kappa_{1} R \approx 100\right)$ particles with $\theta=90^{\circ}$ within linear and nonlinear PB theory. As shown by panel (a) and its inset, the force increases $\propto \sqrt{R}$ with increasing $R$ and decays exponentially with increasing separation $\kappa_{1} L$. Panels (b) and (c) suggest that, for equally sized cylinders, the force increases significantly with decreasing contact angle $\theta$ only within an interval around $90^{\circ}$. Outside this interval the force remains de facto constant and the interval of $\theta$, across which the force actually varies, widens upon decreasing $\kappa_{1} R$. For unequally sized cylinders, if the size asymmetry is moderate, the force may increase as well as decrease if the contact angle deviates from $90^{\circ}$ (panel (d)). However, if the size contrast is high, the force becomes weaker once $\theta$ is slightly shifted away from $90^{\circ}$ in either direction (panel (e)). From panel ( $f$ ) and the inset therein one can infer that the discrepancy between the linear and the nonlinear results diminishes with increasing $\theta$. 
2. Cylinders. For macroscopically long, parallel cylinders, the expressions for the lateral force contributions, expressed per length $L_{y}$ in the $y$-direction, are given by:

$$
\begin{aligned}
\frac{F_{1}(L)}{L_{y}}= & \sum_{i=1}^{3} a_{i} \sqrt{\frac{\pi b_{i}}{\left(\frac{2}{R_{1}}+\frac{2}{R_{2}}\right)}} \exp \left\{-b_{i}\left(L+\frac{\left(D_{1}-D_{2}\right)^{2}}{2\left(R_{1}+R_{2}\right)}\right)\right\} \\
& \times\left[1-\operatorname{erf}\left(\left(D_{1} R_{2}+D_{2} R_{1}\right) \sqrt{\frac{b_{i}}{2 R_{1} R_{2}\left(R_{1}+R_{2}\right)}}\right)\right],
\end{aligned}
$$

$$
\begin{aligned}
\frac{F_{2}(L)}{L_{y}}= & \sum_{i=1}^{3} c_{i} \sqrt{\frac{\pi d_{i}}{\left(\frac{2}{R_{1}}+\frac{2}{R_{2}}\right)}} \exp \left\{-d_{i}\left(L+\frac{\left(D_{1}-D_{2}\right)^{2}}{2\left(R_{1}+R_{2}\right)}\right)\right\} \\
& \times\left[1+\operatorname{erf}\left(\left(D_{1} R_{2}+D_{2} R_{1}\right) \sqrt{\frac{d_{i}}{2 R_{1} R_{2}\left(R_{1}+R_{2}\right)}}\right)\right],
\end{aligned}
$$

and

$$
\frac{F_{3}(L)}{L_{y}}=\sum_{i=1}^{4} g_{i} h_{i} \exp \left\{-h_{i}\left(L+\frac{D_{1}^{2}}{2 R_{1}}+\frac{D_{2}^{2}}{2 R_{2}}\right)\right\} .
$$

We note that for geometrical reasons these expressions are slightly different from those obtained for spheres in eqn (4)-(6). In particular, the contact lines for cylinders are just straight lines and in order to obtain $F_{3}(L)$ there is no need to use the DA. Fig. 3 shows the variation of the $z$-component of the total force $F(L)=F_{1}(L)+F_{2}(L)+F_{3}(L)$, per length $L_{y}$ in the $y$-direction and in units of $10^{2} \kappa_{1}^{2} / \beta$, which the left cylinder exerts on the right one as function of the scaled separation $\kappa_{1} L$ for the sizes $R_{1}$ and $R_{2}$ and for the contact angle $\theta$. Except for a few features, the findings are qualitatively the same as those obtained for spheres in Fig. 2. For example, for $R_{1}=R_{2}=R$ and $\theta=90^{\circ}$, the force between two cylinders also decays exponentially with varying separation $L$ between them and increases with increasing size $R$, but for the cylinders the increase is proportional to $\sqrt{R}$; see Fig. 3(a) and the inset therein. This is evident from the prefactors of the exponential functions in eqn (9) and (10). The variation of the force with respect to $\theta$ as well as eqn (7) and (8) remain the same as for the spheres, because the $\theta$-dependent terms in eqn (9) and (10) have exactly the same form as in eqn (4) and (5). This behavior is confirmed by Fig. 3(b-e). Finally, the comparison of the effective force for $\theta=90^{\circ}$ within linearized and nonlinear PB theory reveals a significant discrepancy between the predictions of the two approaches, which becomes smaller for larger contact angles $\theta$, i.e., as the portion of the particles, dipped into the more polar phase, decreases (see Fig. 3(f)).

\section{B. Water-octanol interface}

Water and lutidine, which are immiscible for sufficiently high temperatures, form a special system in that the bulk properties, i.e., the relative permittivities and the bulk ionic strengths, and consequently the Debye screening lengths, are not very different for the two fluid phases. In contrast to that, in the present subsection we consider another system with silica particles trapped at a water-octanol interface. At room temperature $T=300 \mathrm{~K}$ these two fluids differ starkly with respect to their bulk properties with $\varepsilon_{\mathrm{r}, 1}=80$ for water and $\varepsilon_{\mathrm{r}, 2}=10.3$ for octanol. The partitioning of ions at such an interface leads to highly contrasting bulk ionic strengths: for $I_{1}=10 \mathrm{mM}$ one has $I_{2}=2.9 \times 10^{-3} \mathrm{mM}$; the corresponding resulting Donnan potential equals $3.8 k_{\mathrm{B}} T / e .^{43,44}$ Under these conditions, the inverse Debye length in the water phase is $\kappa_{1} \approx 0.324 \mathrm{~nm}^{-1}$ and the one in the oil phase (octanol) is $\kappa_{2} \approx 0.015 \mathrm{~nm}^{-1}$. The magnitude of the surface charge densities in contact with the two fluid phases also differ significantly; we consider $\sigma_{1}=0.01 e \mathrm{~nm}^{-2}$ and $\sigma_{2}=0.0005 e \mathrm{~nm}^{-2} \cdot{ }^{11,12,45}$

The resulting interactions between the particles are shown in Fig. 4 for a pair of spheres (panels (a) and (c)) as well as for a pair of cylinders (panels (b) and (d)). From Fig. 4(a and b) one can infer that the total force $F(L)$ between equally sized particles increases with increasing radii $\left(R_{1}=R_{2}=R\right)$, both for spheres and cylinders. Whereas for spheres this increase is linear in the particle size (see the inset in Fig. 4(a)), in the case of cylinders it scales $\propto \sqrt{R}$ (see the inset in Fig. 4(b)), which is evident from the data collapse in the insets. Although the line interaction becomes relatively more important in the case of the wateroctanol system - due to a greater mismatch of the system parameters (ionic strengths, permittivities, and charge densities) compared to those of the water-lutidine system - these findings suggest that for micron-sized particles the interaction is still dominated by the surface parts. Fig. 4(c and d) show the variation of the inter-particle forces $F(L)$ as function of the wetting contact angle $\theta$ for spheres and cylinders, respectively. At very short separations, the force varies only within a narrow interval $85^{\circ} \lesssim \theta \lesssim 95^{\circ}$. However, at relatively large separations it varies within a wider interval $75^{\circ} \lesssim \theta \lesssim 105^{\circ}$ of the contact angle. These findings are also in accordance with eqn (7) and (8). For the system considered here, eqn (7) predicts that $F_{1}(L)$ varies appreciably within the interval $86^{\circ} \lesssim \theta \lesssim 94^{\circ}$ whereas, according to eqn (8), $F_{2}(L)$ varies within the interval $71^{\circ} \lesssim \theta \lesssim 109^{\circ}$. At very short separations, the total force is dominated by the surface contribution in medium " 1 " (aqueous phase) due to higher surface charge densities at the particle surfaces. Therefore, if $\theta$ is decreased from $90^{\circ}$, i.e., when the particles become increasingly hydrophilic, the force increases, followed by saturation at around $\theta=85^{\circ}$, as predicted by eqn (7). On the other hand, if $\theta$ is increased beyond $90^{\circ}$ the particles become more hydrophobic. Up to $\theta \approx 95^{\circ}$, for which $F_{1}$ vanishes, the total force decreases as $F_{1}$ decreases. Beyond that, a slight increase of the total force is observed due to $F_{2}$ which, as predicted by eqn (8), increases up to $\theta \approx 109^{\circ}$. As the separation between the particles is increased, in medium " 1 " the interaction decays very fast due to a strong screening by the higher amount of salt present. Consequently, at relatively large separations the total force is dominated by the surface contribution in medium " 2 " (oil phase) and, within the interval $75^{\circ} \lesssim \theta \lesssim 105^{\circ}$, it increases monotonically with increasing contact angle. It is important to note that eqn (7) and (8) are derived by using the fact that the 

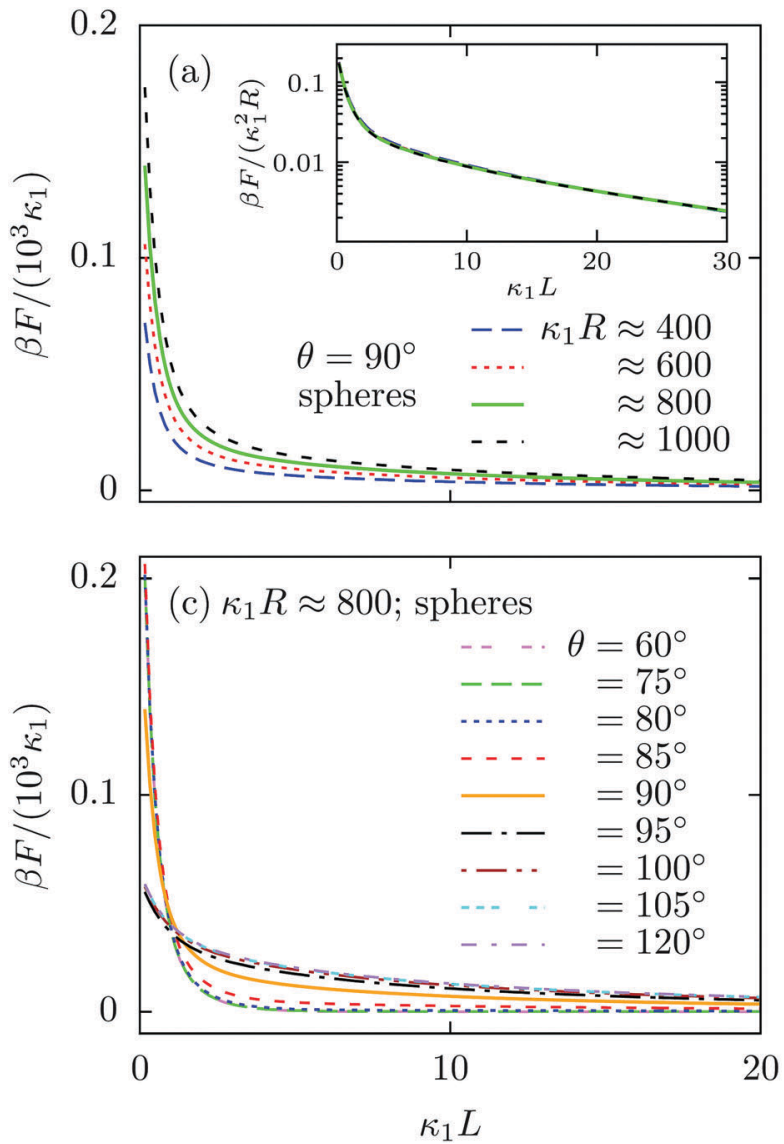
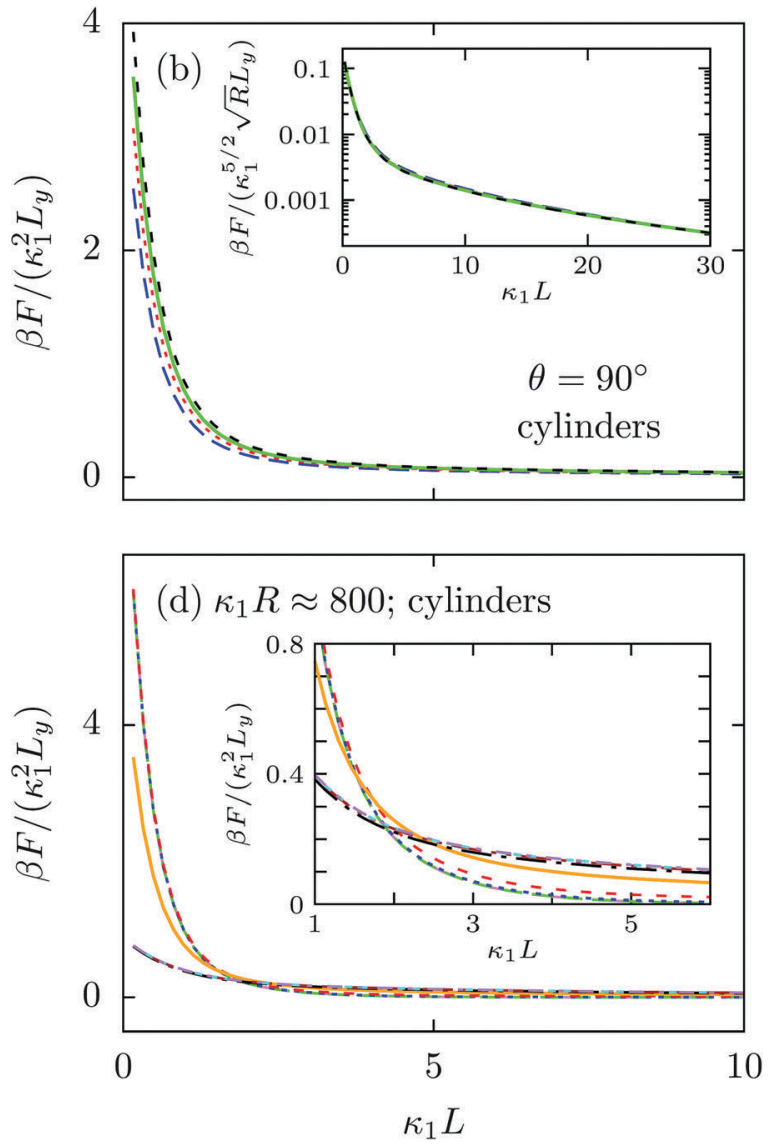

Fig. 4 Panel (a): Variation of the lateral component of the force $F(L)$, expressed in units of $10^{3} \kappa_{1} / \beta$, due to electrostatic interaction between a pair of equally sized $\left(R_{1}=R_{2}=R\right)$ spheres of varying radius with contact angle $90^{\circ}$ as function of their scaled separation $\kappa_{1} L$. The force increases linearly with increasing size of the particles which is evident from the data collapse in the inset. Panel (b): Variation of the $z$-component (see Fig. 1) of the force $F(L)$, per length $L_{y}$ in the $y$-direction, expressed in units of $\kappa_{1}^{2} / \beta$, due to electrostatic interaction between a pair of equally sized cylinders of varying radius with contact angle $90^{\circ}$, as function of their scaled separation $\kappa_{1} L$. Contrary to what one observes for spheres, the force between cylinders is proportional to $\sqrt{R}$. Panel (c): Variation of the lateral component of the force $F(L)$, expressed in units of $10^{3} \kappa_{1} / \beta$, due to electrostatic interaction between a pair of equally sized $\left(\kappa_{1} R \approx 800\right)$ spheres with various contact angles $\theta$, as function of their scaled separation $\kappa_{1} L$. Panel (d): Variation of the $z$-component of the force $F(L)$, per length $L_{y}$ in the $y$-direction, expressed in units of $\kappa_{1}^{2} / \beta$, due to electrostatic interaction between a pair of equally sized $\left(\kappa_{1} R \approx 800\right)$ cylinders for various contact angles $\theta$, as function of their scaled separation $\kappa_{1} L$. Both for spheres and cylinders the force varies within a narrow interval of the contact angle at very short separations. The force increases if the particles are more hydrophilic within this interval of $\theta$. At relatively large separations, however, this interval slightly broadens but the force increases if the particles become more hydrophobic.

error function $\operatorname{erf}(x)$ saturates for $|x| \gtrsim 2$, with the most significant variation occurring only for $|x| \lesssim 1$.5. Therefore, the variation of $F_{2}(L)$ within the intervals $71^{\circ} \lesssim \theta \lesssim 75^{\circ}$ and $105^{\circ} \lesssim \theta \lesssim 109^{\circ}$ are very slow and hardly visible. Since here the silica particles are considered to be weakly charged, the discrepancy between the linear and the nonlinear PB theories become less significant. Still, the forces within the two approaches differ by a factor of 2 even at separations $\kappa_{1} L \approx 10$ for $\theta=90^{\circ}$.

\section{Conclusions}

To conclude, by using the Derjaguin approximation and a fitting procedure for numerical results for the effective interaction between parallel, planar surfaces in contact with two demixed fluids in between, we have calculated the force due to the electrostatic interaction between pairs of spheres or cylinders at close distance from each other at a fluid-fluid interface.
The comparison between the results obtained within linear and nonlinear PB theory shows that the former overestimates the force both for spheres and for cylinders, even at distances of several Debye lengths. Concerning the results within the nonlinear theory, we have investigated the effects of varying the sizes and the contact angle of the particles. Our general study is applicable also to pairs of particles which differ in size. For equally-sized spheres and cylinders the force always decays exponentially with increasing separation, and it scales $\propto R$ for spheres and $\propto \sqrt{R}$ for cylinders, where $R$ is the common radius of the particles. Importantly, for equally-sized particles (both spherical and cylindrical) we have found an interval around the contact angle of $90^{\circ}$, beyond which the force de facto does not vary. We have also obtained simple relations (eqn (7) and (8)) involving the Debye lengths of the two media and the radii of the particles for calculating the width of this interval. These robust results can be expected to be useful for describing 
more general or complex particle interactions at fluid interfaces, which is important for various application perspectives of such systems.

\section{Conflicts of interest}

There are no conflicts to declare.

\section{Acknowledgements}

Open Access funding provided by the Max Planck Society.

\section{References}

1 W. Ramsden, Proc. R. Soc. London, 1903, 72, 156.

2 B. P. Binks and T. S. Horozov, Colloidal Particles at Liquid Interfaces, Cambridge University Press, Cambridge, 2006.

3 S. U. Pickering, J. Chem. Soc., Trans., 1907, 91, 2001.

4 A. D. Dinsmore, M. F. Hsu, M. G. Nikolaides, M. Márquez, A. R. Bausch and D. A. Weitz, Science, 2002, 298, 1006.

5 M. Li, R. L. Harbron, J. V. M. Weaver, B. P. Binks and S. Mann, Nat. Chem., 2013, 5, 529.

6 A. Böker, J. He, T. Emrick and T. P. Russell, Soft Matter, 2007, 3, 1231.

7 B. M. Rey, R. Elnathan, R. Ditcovski, K. Geisel, M. Zanini, M. A. Fernandez-Rodriguez, V. V. Naik, A. Frutiger, W. Richtering, T. Ellenbogen, N. H. Voelcker and L. Isa, Nano Lett., 2016, 16, 157.

8 T. Young, Philos. Trans. R. Soc. London, 1805, 95, 65.

9 P. G. de Gennes, Rev. Mod. Phys., 1985, 57, 827.

10 R. Aveyard, B. P. Binks and J. H. Clint, Adv. Colloid Interface Sci., 2003, 100, 503.

11 T. S. Horozov, R. Aveyard, J. H. Clint and B. P. Binks, Langmuir, 2003, 19, 2822.

12 T. S. Horozov, R. Aveyard, B. P. Binks and J. H. Clint, Langmuir, 2005, 21, 7405.

13 D. Stamou, C. Duschl and D. Johannsmann, Phys. Rev. E, 2000, 62, 5263.

14 P. A. Kralchevsky and K. Nagayama, Adv. Colloid Interface Sci., 2000, 85, 145.

15 M. Oettel, A. Domínguez and S. Dietrich, Phys. Rev. E, 2005, 71, 051401.

16 J. C. Loudet, A. M. Alsayed, J. Zhang and A. G. Yodh, Phys. Rev. Lett., 2005, 94, 018301.

17 M. Oettel and S. Dietrich, Langmuir, 2008, 24, 1425.

18 L. Parolini, A. D. Law, A. Maestro, D. M. A. Buzza and P. Cicuta, J. Phys.: Condens. Matter, 2015, 27, 194119.

19 S. E. Anachkov, I. Lesov, M. Zanini, P. A. Kralchevsky, N. A. Denkov and L. Isa, Soft Matter, 2016, 12, 7632.

20 P. Pieranski, Phys. Rev. Lett., 1980, 45, 569.

21 A. J. Hurd, J. Phys. A: Math. Gen., 1985, 18, L1055.
22 A. Majee, M. Bier and S. Dietrich, J. Chem. Phys., 2014, 140, 164906.

23 Z. Lian, J. Chem. Phys., 2016, 145, 014901.

24 A. Majee, T. Schmetzer and M. Bier, Phys. Rev. E, 2018, 97, 042611.

25 A. Majee, M. Bier and S. Dietrich, J. Chem. Phys., 2016, 145, 064707.

26 S. G. Booth and R. A. W. Dryfe, J. Phys. Chem. C, 2015, 119, 23295.

27 W. B. Russell, D. A. Saville and W. R. Schowalter, Colloidal Dispersions, Cambridge University Press, Cambridge, 1989.

28 M. P. Lyne, B. D. Bowen and S. Levine, J. Colloid Interface Sci., 1992, 150, 374.

29 F. Martínez-López, M. A. Cabrerizo-Vílchez and R. HidalgoÁlvarez, J. Colloid Interface Sci., 2000, 232, 303.

30 R. Aveyard, J. H. Clint, D. Nees and V. N. Paunov, Langmuir, 2000, 16, 1969.

31 R. Aveyard, B. P. Binks, J. H. Clint, P. D. I. Fletcher, T. S. Horozov, B. Neumann, V. N. Paunov, J. Annesley, S. W. Botchway, D. Nees, A. W. Parker, A. D. Ward and A. N. Burgess, Phys. Rev. Lett., 2002, 88, 246102.

32 B. J. Park, J. P. Pantina, E. M. Furst, M. Oettel, S. Reynaert and J. Vermant, Langmuir, 2008, 24, 1686.

33 P. Gao, X. C. Xing, Y. Li, T. Ngai and F. Jin, Sci. Rep., 2014, 4, 4778.

34 C. P. Kelleher, A. Wang, G. I. Guerrero-García, A. D. Hollingsworth, R. E. Guerra, B. J. Krishnatreya, D. G. Grier, V. N. Manoharan and P. M. Chaikin, Phys. Rev. E, 2015, 92, 062306.

35 A. Majee, M. Bier and R. Podgornik, Soft Matter, 2018, 14, 985.

36 V. S. Bagotsky, Fundamentals of Electrochemistry, Wiley, Hoboken, NJ, 2006.

37 R. W. Rampolla and C. P. Smyth, J. Am. Chem. Soc., 1958, 80, 1057.

38 P. D. Gallagher, M. L. Kurnaz and J. V. Maher, Phys. Rev. A: At., Mol., Opt. Phys., 1992, 46, 7750.

39 C. A. Grattoni, R. A. Dawe, C. Y. Seah and J. D. Gray, J. Chem. Eng. Data, 1993, 38, 516.

40 H. D. Inerowicz, W. Li and I. Persson, J. Chem. Soc., Faraday Trans., 1994, 90, 2223.

41 D. R. Lide, Handbook of Chemistry and Physics, CRC, Boca Raton, 82nd edn, 2001-2002.

42 M. Bier, A. Gambassi and S. Dietrich, J. Chem. Phys., 2012, 137, 034504.

43 R. Gulaboski, V. Mirčeski and F. Scholz, Electrochem. Commun., 2002, 4, 277.

44 F. Quentel, V. Mirčeski and M. L'Her, J. Solid State Electrochem., 2008, 12, 31.

45 K. D. Danov, P. A. Kralchevsky and M. P. Boneva, Langmuir, 2004, 20, 6139.

46 Handbook of Mathematical Functions, ed. M. Abramowitz and I. A. Stegun, Dover, New York, 1964. 